\title{
Theory of fault-tolerant quantum computation
}

\author{
Daniel Gottesman* \\ California Institute of Technology, Pasadena, California 91125 \\ and Los Alamos National Laboratories, Los Alamos, New Mexico 87545
}

(Received 18 February 1997)

\begin{abstract}
In order to use quantum error-correcting codes to improve the performance of a quantum computer, it is necessary to be able to perform operations fault-tolerantly on encoded states. I present a theory of fault-tolerant operations on stabilizer codes based on symmetries of the code stabilizer. This allows a straightforward determination of which operations can be performed fault-tolerantly on a given code. I demonstrate that fault-tolerant universal computation is possible for any stabilizer code. I discuss a number of examples in more detail, including the five-quantum-bit code. [S1050-2947(98)06501-9]
\end{abstract}

PACS number(s): 03.67.Lx, 03.65.Bz, 89.80.+h

The development of quantum error-correcting codes [1-6] has stirred great hopes for conquering errors and decoherence in quantum computers. However, just the existence of codes, even very good codes, is not sufficient. It is also necessary to be able to perform operations on encoded states without a catastrophic spread of existing errors. However, until now, fault-tolerant implementations of a universal set of gates were only known for a few codes [7-9].

While most known quantum error-correcting codes can be described using the stabilizer formalism [4-6], there is no similar broadly applicable theory of fault-tolerant operations. A quantum gate, unlike a classical gate, can cause errors to spread both forwards and backwards through the gate. The goal of fault-tolerant operations is to prevent the spread of errors within a block, which could change a single correctable error into two errors, which is perhaps more than the code could handle. Even if we use codes that correct more than one error, the spread of errors within a block rapidly reduces the code's tolerance for errors. Therefore, I define a fault-tolerant operation to be one for which a single operational error can only produce one error within a single encoded block. The assumption is that storage errors on different qubits are independent and that gate errors can only affect quantum bits (qubits) which interact via that gate.

A transversal operation, in which the operation acts independently on each qubit in the block, is a prototypical faulttolerant operation. For instance, a bitwise controlled NOT operation (i.e., $|i\rangle|j\rangle \rightarrow|i\rangle|i \oplus j\rangle$ ) from one block to another is fault-tolerant, since errors can spread only between corresponding qubits in the two blocks.

Unfortunately, for most codes, only a few transversal operations will map one valid codeword to another. I show below that a bitwise operation will transform the stabilizer of a code. If the stabilizer is rearranged, but otherwise left unchanged, the operation will take codewords to codewords. This will give us a few basic operations on various codes with which to start our analysis.

In the quest to perform universal quantum computation, we are not limited to unitary operations. We can also perform measurements. In Sec. III, I analyze the behavior of

*Electronic address: gottesma@t6-serv.lanl.gov certain states when a measurement is made. This allows us to see what operations we can derive from the basic operations by using ancillas and making partial measurements of the state. Ultimately, this will allow us to perform universal computation on any stabilizer code. I also present a more detailed analysis of the five-qubit code, a class of distance two codes, and the code encoding three qubits in eight qubits.

Throughout this paper, I will confine my attention to stabilizer codes. The results can be extended to stabilizer codes over $k$-state systems instead of two-state qubits, but most of the results do not apply to nonstabilizer codes at all. I will indicate those results which do apply more generally as they are presented.

\section{ENCODED NOT AND PHASE}

Before I advance into the full theory of fault-tolerant operations, I will discuss how to perform encoded NOT and phase gates on any stabilizer code. The behavior of these gates under more general transformations will tell us what those transformations actually do to the encoded states.

The stabilizer $S$ of a code is an Abelian subgroup of the group $\mathcal{G}$ generated by the operations

$$
I=\left(\begin{array}{ll}
1 & 0 \\
0 & 1
\end{array}\right), \quad X=\left(\begin{array}{ll}
0 & 1 \\
1 & 0
\end{array}\right), \quad Z=\left(\begin{array}{cc}
1 & 0 \\
0 & -1
\end{array}\right),
$$

and

$$
Y=X \cdot Z=\left(\begin{array}{cc}
0 & -1 \\
1 & 0
\end{array}\right)
$$

acting on each of the $n$ qubits of the code. I will sometimes write $\mathcal{G}_{n}$ to explicitly indicate the number of qubits acted on. The codewords of the code are the states $|\psi\rangle$ which are left fixed by every operator in $S$ and the coding space is the set of such states. Operators in $\mathcal{G}$ which anticommute with some operator in $S$ will take codewords from the coding space into some orthogonal space. By making a measurement to distinguish the various orthogonal spaces, we can then determine 
what error has occurred and correct it. A quantum code encoding $k$ qubits in $n$ qubits will have a stabilizer with $n-k$ generators.

However, there are, in general, a number of operators in $\mathcal{G}$ that commute with all of the operators in $S$. The set of such operators is the normalizer $N(S)$ of $S$ in $\mathcal{G} .{ }^{1} S$ is itself contained in the normalizer, but in general the normalizer is larger than just $S$. If $S$ contains $2^{n-k}$ operators (so it has $n$ $-k$ generators), the normalizer will be generated by $n+k$ operators. In the terminology of Calderbank et al. [5,6], $N(S)$ is the dual code $S^{\perp}$. The set $N(S) / S$ is the set of errors the code cannot detect.

The elements of the normalizer will change one codeword to another, and therefore have a natural interpretation in terms of encoded operations on the code words. Suppose we extend the stabilizer into a maximal set of $n$ commuting operators by choosing $k$ independent commuting operators $\overline{Z_{1}}, \ldots, \overline{Z_{k}}$ from $N(S) / S$. Then consider those codewords which, besides being +1 eigenvectors of the stabilizer generators, are also eigenvectors of $\overline{Z_{1}}, \ldots, \overline{Z_{k}}$. These codewords will be the basis codewords for our code, defining the encoded $|0 \cdots 00\rangle,|0 \cdots 01\rangle, \ldots,|1 \cdots 11\rangle$. The state which has eigenvalue +1 for every $\overline{Z_{i}}$ will be the encoded $|0 \cdots 0\rangle$, the state which has eigenvalue -1 for $\overline{Z_{1}}$ and eigenvalue +1 for the other $\overline{Z_{i}}$ s will be the encoded $|0 \cdots 01\rangle$, and so on. With this definition, $\bar{Z}_{i}$ acts as the encoded $Z$ operator on the $i$ th encoded qubit. If there is just one encoded qubit, I will write $\bar{Z}$ instead of $\overline{Z_{1}}$.

Now, the remaining elements of $N(S)$ will not commute with all of the encoded $Z$ operators. We can complete the set of generators for $N(S)$ by choosing $k$ additional operators $\overline{X_{i}}(i=1, \ldots, k)$ such that $\overline{X_{i}}$ commutes with $\overline{Z_{j}}$ when $i \neq j$, but $\overline{X_{i}}$ anticommutes with $\overline{Z_{i}} \cdot \overline{X_{i}}$ is then just the encoded bit flip operator on the $i$ th encoded qubit. Again, I write $\bar{X}$ when there is just one encoded qubit. An arbitrary element of $N(S)$ is some other encoded operation and can be written as the product of $\bar{X}$ s and $\bar{Z}$ s. If two elements of $N(S)$ differ by an element of the stabilizer, they act the same way on any code word (since the stabilizer element just fixes the codeword). Therefore the actual set of encoded operations represented in $N(S)$ is $N(S) / S$.

DiVicenzo and Shor showed how to perform syndrome measurement and error correction fault-tolerantly on any stabilizer code [10]. Using the same methods, we can measure the eigenvalue of any operator in $\mathcal{G}$, even if it is not in $S$. This also enables us to prepare the encoded zero state of any stabilizer code by performing error correction and measuring the eigenvalue of the $\bar{Z}$ operators.

\section{MORE GENERAL OPERATIONS}

So far, we have only considered applying products of $X$, $Y$, and $Z$ to the codewords. However, this is not the most general thing we could do. Suppose we have some totally

\footnotetext{
${ }^{1}$ Strictly speaking, this is the centralizer of $S$, but in this case it is equal to the normalizer, since $G^{-1} M G= \pm G^{-1} G M= \pm M$, and not both $M$ and $-M$ are in $S$.
}

arbitrary unitary transformation $U$ we wish to apply to our codewords. How does this affect other operators, such as the elements of $S$ and $N(S)$ ?

$$
U M|\psi\rangle=U M U^{\dagger} U|\psi\rangle
$$

so $|\psi\rangle$ is an eigenvector of $M$ if and only if $U|\psi\rangle$ is an eigenvector of $U M U^{\dagger}$. Furthermore, they have the same eigenvalue. Thus, by applying $U$ to $|\psi\rangle$, we effectively transform any operator $M$ of interest into $U M U^{\dagger}$ (this fact is also true for nonstabilizer codes). In order for the state $|\psi\rangle$ to remain a codeword, the state $U|\psi\rangle$ must still be in the coding space, so $U M U^{\dagger}$ must also fix all the codewords $|\psi\rangle$ for every $M \in S$. Let us consider a restricted set of possible $U^{\prime}$ 's, those for which $U M U^{\dagger}$ is actually in $\mathcal{G}$ [so $\mathrm{U}$ is in the normalizer $N(\mathcal{G})$ of $\mathcal{G}$ in $U(n)] . N(\mathcal{G})$ is generated by Hadamard rotations, $\pi / 2$ phase rotations, and controlled NOT operations [5,11] (a proof of this fact appears in Appendix A). Calderbank et al. call $N(\mathcal{G})$ the Clifford group. By the definition of the stabilizer and the coding space, we need $U M U^{\dagger}$ to actually be in $S$ for all $M \in S$. Therefore, $U$ is actually in the normalizer of $S$ in $U(n)$. The same criterion was found previously by Knill [12]. Note that the normalizer of $S$ in $U(n)$ is not necessarily a subset of $N(\mathcal{G})$.

When we restrict our attention to operations that are in both the normalizer of $\mathcal{G}$ in $U(n)$ and the normalizer of $S$ in $U(n)$, it becomes straightforward to determine the operation actually performed on the encoded states. First, note that the $\bar{X}$ and $\bar{Z}$ operators transform into operators that also commute with everything in $S$. Thus, we can rewrite them as products of the original $\bar{X}$ 's, $\bar{Z}$ 's, and elements of $S$. The elements of $S$ just give us the equivalence between elements of $N(S)$ discussed in Sec. I, so we have deduced a transformation of the encoded $X$ and $Z$ operators. Furthermore, we know this encoded transformation also lies in the normalizer of $\mathcal{G}_{k}$.

Typically, we want to consider transversal operations $U$, which are equal to the tensor product of single-qubit operations (or operations that only affect one qubit per block). For the moment, we will only consider operations of this form and see what collections of them will do to the stabilizer. Before launching into an analysis of which gates can be used on which codes, I will present an overview of the gates that are amenable to this sort of analysis.

For instance, one of the simplest and most common faulttolerant operations is the Hadamard rotation

$$
R=\frac{1}{\sqrt{2}}\left(\begin{array}{cc}
1 & 1 \\
1 & -1
\end{array}\right) .
$$

Let us see what this does to $X, Y$, and $Z$.

$$
\begin{gathered}
R X R^{\dagger}=\frac{1}{2}\left(\begin{array}{cc}
1 & 1 \\
1 & -1
\end{array}\right)\left(\begin{array}{cc}
1 & -1 \\
1 & 1
\end{array}\right)=\left(\begin{array}{cc}
1 & 0 \\
0 & -1
\end{array}\right)=Z, \\
R Z R^{\dagger}=\frac{1}{2}\left(\begin{array}{cc}
1 & 1 \\
1 & -1
\end{array}\right)\left(\begin{array}{cc}
1 & 1 \\
-1 & 1
\end{array}\right)=\left(\begin{array}{ll}
0 & 1 \\
1 & 0
\end{array}\right)=X, \\
R Y R^{\dagger}=\frac{1}{2}\left(\begin{array}{cc}
1 & 1 \\
1 & -1
\end{array}\right)\left(\begin{array}{cc}
-1 & 1 \\
1 & 1
\end{array}\right)=\left(\begin{array}{cc}
0 & 1 \\
-1 & 0
\end{array}\right)=-Y .
\end{gathered}
$$


Therefore, applying $R$ bitwise will switch all the $X$ 's and all the $Z$ 's, and give a factor of -1 for each $Y$. If we do this to the elements of the stabilizer and get other elements of the stabilizer, this is a valid fault-tolerant operation. The sevenqubit code is an example of a code for which this is true.

Another common bitwise operation is the $i$ phase:

$$
P=\left(\begin{array}{ll}
1 & 0 \\
0 & i
\end{array}\right) \text {. }
$$

On the basic operations $X, Y$, and $Z$ it acts as follows:

$$
\begin{gathered}
P X P^{\dagger}=\left(\begin{array}{ll}
1 & 0 \\
0 & i
\end{array}\right)\left(\begin{array}{cc}
0 & -i \\
1 & 0
\end{array}\right)=\left(\begin{array}{cc}
0 & -i \\
i & 0
\end{array}\right)=i Y, \\
P Y P^{\dagger}=\left(\begin{array}{ll}
1 & 0 \\
0 & i
\end{array}\right)\left(\begin{array}{cc}
0 & i \\
1 & 0
\end{array}\right)=\left(\begin{array}{cc}
0 & i \\
i & 0
\end{array}\right)=i X, \\
P Z P^{\dagger}=\left(\begin{array}{ll}
1 & 0 \\
0 & i
\end{array}\right)\left(\begin{array}{ll}
1 & 0 \\
0 & i
\end{array}\right)=\left(\begin{array}{cc}
1 & 0 \\
0 & -1
\end{array}\right)=Z .
\end{gathered}
$$

This switches $X$ and $Y$, but with extra factors of $i$, so there must be a multiple of $4 X^{\prime}$ 's and $Y$ 's for this to be a valid operation. Again, the seven-qubit code is an example of one where it is. Note that a factor of $i$ appears generically in any operation that switches $Y$ with $X$ or $Z$, because $Y^{2}=-1$, while $X^{2}=Z^{2}=+1$. The operations in $N(\mathcal{G})$ actually permute $\sigma_{X}=X, \sigma_{Z}=Z$, and $\sigma_{Y}=i Y$, but for consistency with earlier publications I have retained the notation of $X, Y$, and $Z$. The most general single qubit operation in $N(\mathcal{G})$ can be viewed as a rotation of the Bloch sphere permuting the three coordinate axes.

We can also consider two-qubit operations, such as the controlled NOT. Now we must consider transformations of the two involved blocks combined. The stabilizer group of the two blocks is $S \times S$, and we must see how the basic operations $X \otimes I, Z \otimes I, I \otimes X$, and $I \otimes Z$ transform under the proposed operation. In fact, we will also need to know the transformation of $X \otimes Y$ and other such operators, but the transformation induced on $\mathcal{G} \times \mathcal{G}$ is a group homomorphism, so we can determine the images of everything from the images of the four elements listed above.

It is straightforward to show that the controlled NOT induces the following transformation:

$$
\begin{aligned}
& X \otimes I \rightarrow X \otimes X, \\
& Z \otimes I \rightarrow Z \otimes I, \\
& I \otimes X \rightarrow I \otimes X, \\
& I \otimes Z \rightarrow Z \otimes Z .
\end{aligned}
$$

It is easy to see here how amplitudes are copied forwards and phases are copied backwards. The transformation laws for $R$, $P$, and CNOT are also given in [5].

There are a number of basic gates in $N(\mathcal{G})$ beyond the ones given above. As with the examples above, any gate can be characterized by its transformation of the generators of $\mathcal{G}$ (or $\mathcal{G} \times \mathcal{G}$ for two-qubit operations, and so on). The primary constraint that must be met is to preserve the algebraic prop- erties of the operators. In fact, there is a complete equivalence between the possible gates and the automorphisms of $D_{4}$ (the group of products of $I, X, Y$, and $Z$ ) or direct products of copies of $D_{4}$ (for multiple-qubit gates) [13].

Given any such automorphism, we first substitute $i Y$ for $Y$ to get the actual transformation. Then we note that $|0\rangle$ is the "encoded zero" for the "code" with stabilizer $\{I, Z\}$. We know how $Z$ transforms under $U$, so $|0\rangle$ transforms to the state fixed by $U Z U^{\dagger}$. In addition, $|1\rangle=X|0\rangle$, so $U|1\rangle$ $=U X U^{\dagger} U|0\rangle$. For instance, consider the cyclic transformation

$$
T=X \rightarrow i Y \rightarrow Z \rightarrow X
$$

Since $Z \rightarrow X$,

$$
|0\rangle \rightarrow 1 / \sqrt{2}(|0\rangle+|1\rangle)
$$

Also, $X \rightarrow i Y$, so

$$
|1\rangle \rightarrow i / \sqrt{2} Y(|0\rangle+|1\rangle)=-i / \sqrt{2}(|0\rangle-|1\rangle) .
$$

Thus, the matrix for $T$ is

$$
T=\frac{1}{\sqrt{2}}\left(\begin{array}{cc}
1 & -i \\
1 & i
\end{array}\right) .
$$

We can perform a similar procedure to determine the matrix corresponding to a multiple-qubit transformation.

The next question of interest is how much have we restricted our computational power by restricting our attention to the normalizer of $\mathcal{G}$ ? Again, the normalizer of $\mathcal{G}$ is exactly the group generated by the Hadamard transform $R$, the phase $P$, and the controlled NOT. Unfortunately, this group alone is of only limited interest. Knill [13] has shown that a quantum computer using only operations from this group can be simulated efficiently on a classical computer. ${ }^{2}$ However, the addition of just the Toffoli gate to this group is sufficient to make the group universal [7].

\section{MEASUREMENTS}

Now I will discuss what happens if we perform a measurement on a stabilizer code. Measuring individual qubits of an actual code is not of great interest, but the results of this section will be quite helpful in determining what can be done by combining measurements and specific fault-tolerant operations. If the computer starts in a partially known state, for instance if the first qubit is $|0\rangle$, it can often be described by a stabilizer. The results of this section are most useful for analyzing the behavior of stabilizers used to describe computers with this sort of initial condition.

\footnotetext{
${ }^{2}$ The argument goes as follows: we start with an $n$-qubit state $|0\rangle$ which is the single state for the stabilizer code $\left\langle Z_{1}, \ldots, Z_{n}\right\rangle$. Each operation transforms the state and the stabilizer as above. We can follow each transformation on a classical computer in $O\left(n^{2}\right)$ steps. A measurement picks at random one of the basis kets in the codeword, which can also be chosen classically [4,14]. This still leaves the question of partial measurement of the full state, but the results of Sec. III show that this can also be classically simulated.
} 
Now, using the method of DiVincenzo and Shor [10], we can measure any operator $A$ in $\mathcal{G}$. There are three possible relationships between $A$ and $S$. First of all, $A$ could actually be in $S$. Then measuring $A$ tells us nothing about the state of the system and does not change it at all. The result of this measurement will always be +1 for a valid codeword. The second possibility is for $A$ to commute with everything in $S$ but not to actually be in $S$. Then $A$ is equivalent to a nontrivial element of $N(S) / S$ and measuring it will give us information about the state of the system. This is usually inadvisable.

The third possibility, that $A$ anticommutes with something in $S$, is the most interesting. In this case, we can choose the generators of $S$ so that $A$ anticommutes with the first generator $M_{1}$ and commutes with the remaining generators $M_{2}, \ldots, M_{n-k}$ (we can do this since if generator $M_{j}$ anticommutes with $A$, we can replace it with $M_{1} M_{j}$, which commutes). Then measuring $A$ does not disturb the eigenvectors of $M_{2}$ through $M_{n-k}$, so they still fix the new state, and are in the new stabilizer. The eigenvectors of $M_{1}$ are disturbed, however, and $M_{1}$ no longer fixes the states. Measuring $A$ applies one of the projection operators $P_{+}$or $P_{-}$, where

$$
P_{ \pm}=\frac{1}{2}(I \pm A)
$$

Then $M_{1}^{\dagger} P_{-} M_{1}=M_{1}^{\dagger} M_{1} P_{+}=P_{+}$, so if $|\psi\rangle$ is some codeword,

$$
M_{1}^{\dagger} P_{-}|\psi\rangle=M_{1}^{\dagger} P_{-} M_{1}|\psi\rangle=P_{+}|\psi\rangle .
$$

If the measurement result is +1 , we do nothing else, and have thus applied $P_{+}$. If the measurement result is -1 , apply $M_{1}^{\dagger}=M_{1}$, resulting in the overall application of $P_{+}$. Either way, the new state is a +1 -eigenvector of $A$. The system is thus in the space with stabilizer generated by $A, M_{2}, \ldots, M_{n-k}$. > From now on, I will often say "measure" when I mean "measure and correct for a result of $-1 .$,

Note that this construction works outside the framework of stabilizer codes. All we really need is a state $|\psi\rangle$, with $M|\psi\rangle=|\psi\rangle$ for some unitary $M$. Then, as above, we can perform the projection $P_{+}$for any operator $A$ satisfying $A^{2}$ $=1$ and $\{M, A\}=0$.

We will want to know just where in the space a given state goes. To do this, look at the elements of $N(S) / S$. If before the measurement, the state is an eigenvector of $N$ $\in N(S)$, it will also be an eigenvector of $N^{\prime}=M N$ for all $M \in S$; that is, for all $N^{\prime}$ in the same coset as $N$ in $N(S) / S$. After measuring $A$, the state will no longer be an eigenvector of $N$ if $N$ anticommutes with $A$, but it will still be an eigenvector of $M_{1} N$, which commutes with $A$. Furthermore, the eigenvalue of $M_{1} N$ stays the same. Therefore, by measuring $A$ (and correcting the state if the result is -1 ), we effectively transform the operator $N$ into $M_{1} N$. We could equally well say it is transformed to $M M_{1} N$ instead, where $M \in S$ commutes with $A$, but this will produce the same transformation of the cosets of $N(S) / S$ to $N\left(S^{\prime}\right) / S^{\prime}$ (where $S^{\prime}$ is the stabilizer after the measurement). Of course, if $N$ commutes with $A$, measuring $A$ leaves $N$ unchanged.
In summary, when we measure an operator $A$, we perform the following procedure on the stabilizer and $\bar{X}$ and $\bar{Z}$ operators.

(1) Identify an element $M_{1} \in S$ that anticommutes with $A$.

(2) Rewrite the remaining generators of $S$ and the $\bar{X}$ and $\bar{Z}$ operators by multiplying by $M_{1}$ if the original operator does not commute with $A$. The rewritten operators are equivalent to the old ones, but now they all commute with $A$.

(3) Replace $M_{1}$ with $A$. We now have the new stabilizer and new $\bar{X}$ and $\bar{Z}$ operators.

Let us see how all this works with a simple, but very useful, example. Suppose we have two qubits, one in an arbitrary state $|\psi\rangle$, the other initialized to $|0\rangle$. The space of possible states then has stabilizer $I \otimes Z$. Suppose we perform a controlled NOT from the first qubit to the second. This transforms the stabilizer to $Z \otimes Z$. Now let us measure the operator $I \otimes i Y$ (we use the factor of $i$ to ensure that the result is \pm 1 ). This anticommutes with $Z \otimes Z$, so if we get +1 , we leave the result alone, and if we get -1 , we apply $Z \otimes Z$ to the state. The new state is in a +1 -eigenstate of $I \otimes i Y$, that is, $|\phi\rangle(|0\rangle+i|1\rangle)$.

How is $|\psi\rangle$ related to $|\phi\rangle$ ? For the original "code," $\bar{X}$ $=X \otimes I$ and $\bar{Z}=Z \otimes I$. After the CNOT, $\bar{X}=X \otimes X$ and $\bar{Z}$ $=Z \otimes I . X \otimes X$ does not commute with $I \otimes i Y$, but the equivalent operator $Y \otimes Y=(X \otimes X)(Z \otimes Z)$ does. $Z \otimes I$ does commute with $I \otimes i Y$, so it stays the same. Since the second qubit is guaranteed to be in the +1 eigenstate of $i Y$, we might as well ignore it. The effective $\bar{X}$ and $\bar{Z}$ operators for the first qubit are thus $-i Y$ and $Z$, respectively. This means we have transformed $\bar{X} \rightarrow-i \bar{X} \bar{Z}$ and $\bar{Z} \rightarrow \bar{Z}$. This is the operation $P^{\dagger}$.

This example is simple enough that it is easy to check:

$$
\begin{aligned}
&|00\rangle \rightarrow|00\rangle=|0\rangle \frac{1}{2}[(|0\rangle+i|1\rangle)+(|0\rangle-i|1\rangle)] \\
& \rightarrow|0\rangle(|0\rangle \pm i|1\rangle) \\
& \rightarrow|0\rangle(|0\rangle+i|1\rangle) \\
&|10\rangle \rightarrow|11\rangle=|1\rangle \frac{i}{2}[-(|0\rangle+i|1\rangle)+(|0\rangle-i|1\rangle)] \\
& \rightarrow i|1\rangle(\mp|0\rangle-i|1\rangle) \\
& \rightarrow \pm i|1\rangle(\mp|0\rangle \mp i|1\rangle)=-i|1\rangle(|0\rangle+i|1\rangle) .
\end{aligned}
$$

Thus, ignoring the second qubit gives $|0\rangle \rightarrow|0\rangle$ and $|1\rangle \rightarrow$ $-i|1\rangle$, which is $P^{\dagger}$.

This result is already quite interesting when coupled with the observation that $P$ and CNOT suffice to produce $R$ as long as we can prepare and measure states in the basis $|0\rangle$ $\pm|1\rangle$ [8]. To do this we start out with the state $|\psi\rangle$ plus an ancilla $|0\rangle+|1\rangle$. Thus, the initial stabilizer is $I \otimes X, \bar{X}=X$ $\otimes I$, and $\bar{Z}=Z \otimes I$. Apply a CNOT from the second qubit to the first. Now the stabilizer is $X \otimes X, \bar{X}=X \otimes I$, and $\bar{Z}=Z$ $\otimes Z$. Apply $P$ to the second qubit, so the stabilizer is $X$ $\otimes i Y, \bar{X}=X \otimes I$, and $\bar{Z}=Z \otimes Z$. Measure $I \otimes X$, performing $X \otimes i Y$ if the result is -1 . This produces $\bar{X}=X \otimes I$ and $\bar{Z}$ 
$=i Y \otimes X$, so dropping the second qubit results in the transformation $Q: X \rightarrow X, Z \rightarrow i Y$. But $R=P Q^{\dagger} P$ :

$$
\begin{gathered}
X \rightarrow i Y \rightarrow Z \rightarrow Z, \\
Z \rightarrow Z \rightarrow-i Y \rightarrow X .
\end{gathered}
$$

Coupled with the previous result, which derives $P$ from CNOT, this allows us to get any single qubit transformation in the normalizer of $\mathcal{G}$ provided we can perform a CNOT operation.

Another interesting application is to gain a new viewpoint on quantum teleportation. Suppose we have three qubits which start in the state $|\psi\rangle(|00\rangle+|11\rangle)$. The initial stabilizer is $I \otimes X \otimes X$ and $I \otimes Z \otimes Z, \bar{X}=X \otimes I \otimes I$, and $\bar{Z}=Z \otimes I \otimes I$. We assume the third qubit is far away, so we can do no operations interacting it directly with the other two qubits. We can, however, perform operations on it conditioned on the result of measuring the other qubits. We begin by performing a CNOT from qubit one to two. The stabilizer is now $I \otimes X$ $\otimes X$ and $Z \otimes Z \otimes Z, \bar{X}=X \otimes X \otimes I$, and $\bar{Z}=Z \otimes I \otimes I$. Measure $X$ for qubit one and discard qubit one. If the measurement result was +1 , we leave the state alone; if it was -1 , we perform $Z$ on qubits two and three. The stabilizer is now $X$ $\otimes X, \bar{X}=X \otimes I$ and $\bar{Z}=Z \otimes Z$. Now measure $Z$ for the new first qubit. If the result is +1 , we leave the final qubit alone; if it is -1 , we apply $X$ to the last qubit. This results in $\bar{X}$ $=X$ and $\bar{Z}=Z$, both acting on the last qubit. We have succesfully teleported the state $|\psi\rangle$. The operations conditioned on measurement results needed for teleportation arise here naturally as the corrections to the stabilizer for alternate measurement results. The formalism would have told us just as easily what operations were necessary if we had begun with a different Bell state or a more complicated entangled state (as long as it can still be described by a stabilizer).

The methods of this section and the previous one will allow us to construct a universal set of gates for any stabilizer code, but they are also useful in a wide variety of other circumstances. As one application, Appendix A gives a proof that $N(\mathcal{G})$ is generated by $R, P$, and CNOT. The above analysis of teleportation is another example. In general, any system involving only measurements and gates from $N(\mathcal{G})$ can be profitably analyzed using these methods.

\section{OPERATIONS ON CSS CODES}

In this section, I will finally begin to look at the problem of which gates can be applied to specific codes. One of the best classes of codes for fault-tolerant computation are the Calderbank-Shor-Steane (CSS) codes [2,3], which are converted from certain classical codes. These codes have a stabilizer which can be written as the direct product of two sectors, one of which is formed purely from $X$ 's and one formed just from $Z$ 's. These two sectors correspond to the two dual classical codes that go into the construction of the code.

Shor [7] showed that a punctured doubly even self-dual CSS code could be used for universal computation. An example of such a code is the seven-qubit code, whose stabilizer is given in Table I. From the stabilizer, we can now understand why such codes allow the fault-tolerant imple-
TABLE I. The stabilizer and encoded $X$ and $Z$ for the sevenqubit code.

\begin{tabular}{llllllll}
\hline \hline$M_{1}$ & $X$ & $X$ & $X$ & $X$ & $I$ & $I$ & $I$ \\
$M_{2}$ & $X$ & $X$ & $I$ & $I$ & $X$ & $X$ & $I$ \\
$M_{3}$ & $X$ & $I$ & $X$ & $I$ & $X$ & $I$ & $X$ \\
$M_{4}$ & $Z$ & $Z$ & $Z$ & $Z$ & $I$ & $I$ & $I$ \\
$M_{5}$ & $Z$ & $Z$ & $I$ & $I$ & $Z$ & $Z$ & $I$ \\
$M_{6}$ & $Z$ & $I$ & $Z$ & $I$ & $Z$ & $I$ & $Z$ \\
$\bar{X}$ & $I$ & $I$ & $I$ & $I$ & $X$ & $X$ & $X$ \\
$\bar{Z}$ & $I$ & $I$ & $I$ & $I$ & $Z$ & $Z$ & $Z$ \\
\hline \hline
\end{tabular}

mention of the Hadamard rotation, the $\pi / 2$ rotation, and the controlled NOT.

The Hadamard rotation switches $X$ and $Z$. For a CSS code, this is a symmetry of the stabilizer if and only if the $X$ sector of the stabilizer is the same as the $Z$ sector. Therefore the two classical codes had to be identical, and the quantum code must be derived from a classical code that contains its own dual. As we can see, this works for the seven-qubit code. In order to understand what the Hadamard rotation does to the encoded states, we must look at what it does to the encoded $X$ and $Z$ operations. For a punctured self-dual CSS code, the $\bar{X}$ and $\bar{Z}$ operations can again be taken to be the same, so the Hadamard rotation will just switch them. It is therefore an operation which switches encoded $X$ with encoded $Z$, and is thus an encoded Hadamard rotation.

Similarly, for a self-dual code, the $\pi / 2$ rotation will convert the $X$ generators into the product of all $Y$ 's. This just converts an $X$ generator into its product with the corresponding $Z$ generator, so this is a valid fault-tolerant operation, provided the overall phase is correctly taken care of. There is a factor of $i$ for each $X$, so there must be a multiple of four $X$ 's in each element of the stabilizer for that to work out in general. This will only be true of a doubly even CSS code, which gives us the other requirement for Shor's methods. Again, we can see that the seven-qubit code meets this requirement. Such a code will have three $\bmod 4 X$ 's in the $\bar{X}$ operation, so the bitwise $\pi / 4$ converts $\bar{X}$ to $-i \bar{Y}$. This is thus an encoded $-\pi / 2$ rotation.

Finally, we get to the controlled NOT. This can be performed bitwise on any CSS code. We must look at its operation on $M \otimes I$ and $I \otimes M$. In the first case, if $M$ is an $X$ generator, it becomes $M \otimes M$. Since both the first and second blocks have the same stabilizer, this is an element of $S \times S$. If $M$ is a $Z$ generator, $M \otimes I$ becomes $M \otimes I$ again. Similarly, if $M$ is an $X$ generator, $I \otimes M$ becomes $I \otimes M$, and if $M$ is a $Z$ generator, $I \otimes M$ becomes $M \otimes M$, which is again in $S \times S$. For an arbitrary CSS code, the $\overline{X_{i}}$ operators are formed from the product of all $X$ 's and the $\bar{Z}_{i}$ operators are formed from the product of all $Z$ 's. Therefore,

$$
\begin{gathered}
\overline{X_{i}} \otimes I \rightarrow \overline{X_{i}} \otimes \overline{X_{i}}, \\
\overline{Z_{i}} \otimes I \rightarrow \overline{Z_{i}} \otimes I, \\
I \otimes \overline{X_{i}} \rightarrow I \otimes \overline{X_{i}},
\end{gathered}
$$


TABLE II. The stabilizer and encoded $X$ and $Z$ for the fivequbit code.

\begin{tabular}{lccccc}
\hline \hline$M_{1}$ & $X$ & $Z$ & $Z$ & $X$ & $I$ \\
$M_{2}$ & $I$ & $X$ & $Z$ & $Z$ & $X$ \\
$M_{3}$ & $X$ & $I$ & $X$ & $Z$ & $Z$ \\
$M_{4}$ & $Z$ & $X$ & $I$ & $X$ & $Z$ \\
$\bar{X}$ & $X$ & $X$ & $X$ & $X$ & $X$ \\
$\bar{Z}$ & $Z$ & $Z$ & $Z$ & $Z$ & $Z$ \\
\hline \hline
\end{tabular}

$$
I \otimes \overline{Z_{i}} \rightarrow \overline{Z_{i}} \otimes \overline{Z_{i}}
$$

Thus, the bitwise CNOT produces an encoded CNOT for every encoded qubit in the block.

In fact, we can now easily prove that codes of the general CSS form are the only codes for which bitwise CNOT is a valid fault-tolerant operation. Let us take a generic element of the stabilizer and write it as $M N$, where $M$ is the product of $X$ 's and $N$ is the product of $Z$ 's. Then under bitwise CNOT, $M N \otimes I \rightarrow M N \otimes M$, which implies $M$ itself is an element of the stabilizer. The stabilizer is a group, so $N$ is also an element of the stabilizer. Therefore, the stabilizer breaks up into a sector made solely from $X$ 's and one made solely from Z's, which means the code is of the CSS type.

\section{THE FIVE-QUBIT CODE}

One code of particular interest is the five-qubit code $[11,15]$, which is the smallest possible code to correct a single error. Until now, there were no known fault-tolerant operations that could be performed on this code except the simple encoded $X$ and encoded $Z$. One presentation [5] of the five-qubit code is given in Table II. This presentation has the advantage of being cyclic, which simplifies somewhat the analysis below.

This stabilizer is invariant under the transformation $T: X \rightarrow i Y \rightarrow Z \rightarrow X$ bitwise. For instance,

$$
M_{1}=X \otimes Z \otimes Z \otimes X \otimes I \rightarrow-Y \otimes X \otimes X \otimes Y \otimes I=M_{3} M_{4} .
$$

By the cyclic property of the code, $M_{2}$ through $M_{4}$ also get transformed into elements of the stabilizer, so this is a valid fault-tolerant operation. It transforms

$$
\bar{X} \rightarrow i \bar{Y} \rightarrow \bar{Z}
$$

Therefore, this operation performed bitwise performs an encoded version of itself. Operations which have this property are particularly useful because they are easy to apply to concatenated codes $[8,9,16]$.

There is no nontrivial two-qubit operation in the normalizer of $\mathcal{G}$ that can be performed transversally on this code. However, there is a three-qubit transformation $T_{3}$ that leaves $S \times S \times S$ invariant:

$$
\begin{gathered}
X \otimes I \otimes I \rightarrow i X \otimes Y \otimes Z \\
Z \otimes I \otimes I \rightarrow i Z \otimes X \otimes Y \\
I \otimes X \otimes I \rightarrow i Y \otimes X \otimes Z,
\end{gathered}
$$

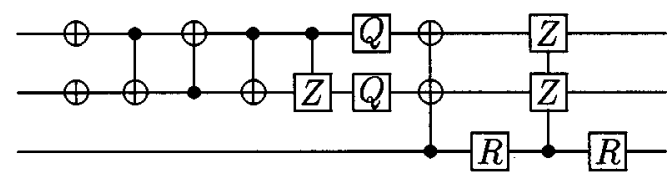

FIG. 1. Network to perform the $T_{3}$ gate.

$$
\begin{gathered}
I \otimes Z \otimes I \rightarrow i X \otimes Z \otimes Y, \\
I \otimes I \otimes X \rightarrow X \otimes X \otimes X, \\
I \otimes I \otimes Z \rightarrow Z \otimes Z \otimes Z .
\end{gathered}
$$

On operators of the form $M \otimes I \otimes I$ or $I \otimes M \otimes I$, this transformation applies cyclic transformations as above to the other two slots. Operators $I \otimes I \otimes M$ just become $M \otimes M \otimes M$, which is clearly in $S \times S \times S$. The matrix of $T_{3}$ is (up to normalization)

$$
T_{3}=\left(\begin{array}{cccccccc}
1 & 0 & i & 0 & i & 0 & 1 & 0 \\
0 & -1 & 0 & i & 0 & i & 0 & -1 \\
0 & i & 0 & 1 & 0 & -1 & 0 & -i \\
i & 0 & -1 & 0 & 1 & 0 & -i & 0 \\
0 & i & 0 & -1 & 0 & 1 & 0 & -i \\
i & 0 & 1 & 0 & -1 & 0 & -i & 0 \\
-1 & 0 & i & 0 & i & 0 & -1 & 0 \\
0 & 1 & 0 & i & 0 & i & 0 & 1
\end{array}\right)
$$

As with $T$, this operation performs itself on the encoded states. A possible network to produce this operation (based on the construction in Sec. III) is given in Fig. 1.

If we add in the possibility of measurements, this threequbit operation along with $T$ will allow us to perform any operation in the normalizer of $\mathcal{G}$. I will describe how to do this on unencoded qubits, and since $T$ and $T_{3}$ bitwise just perform themselves, this will tell us how to do the same operations on the encoded qubits.

To perform $P$, first prepare two ancilla qubits in the state $|00\rangle$ and use the data qubit as the third qubit. The original stabilizer is $Z \otimes I \otimes I$ and $I \otimes Z \otimes I, \bar{X}=I \otimes I \otimes X$, and $\bar{Z}=I$ $\otimes I \otimes Z$. Now apply $T_{3}$, so that the stabilizer is $i Z \otimes X \otimes Y$ and $i X \otimes Z \otimes Y, \bar{X}=X \otimes X \otimes X$, and $\bar{Z}=Z \otimes Z \otimes Z$. Measure $Z$ for the second and third qubits. The resulting $\bar{X}=i Y \otimes I \otimes Z$ and $\bar{Z}=Z \otimes Z \otimes Z$. Dropping the last two qubits, we have $X \rightarrow i Y$ and $Z \rightarrow Z$, which is $P$. Again, $Q=T^{\dagger} P$ and $R=P Q^{\dagger} P$, so we can perform any single qubit operation.

To get a two-qubit operation, prepare a third qubit in the state $|0\rangle$ and apply $T_{3}$. This results in the stabilizer $Z \otimes Z$ $\otimes Z, \overline{X_{1}}=i X \otimes Y \otimes Z, \overline{X_{2}}=i Y \otimes X \otimes Z, \overline{Z_{1}}=i Z \otimes X \otimes Y$, and $\overline{Z_{2}}=i X \otimes Z \otimes Y$. Measure $X$ for the second qubit and throw it out. This leaves the transformation

$$
\begin{aligned}
& X \otimes I \rightarrow i Y \otimes I, \\
& I \otimes X \rightarrow i Y \otimes Z,
\end{aligned}
$$




$$
\begin{aligned}
& Z \otimes I \rightarrow i Z \otimes Y, \\
& I \otimes Z \rightarrow i Y \otimes X .
\end{aligned}
$$

This operation can be produced by applying $Q$ to the second qubit (switching $Z$ and $i Y$ ), then a CNOT from the second qubit to the first one, then $P$ to the first qubit and $T^{2}$ to the second qubit. Therefore, we can also get a CNOT by performing this operation with the appropriate one-qubit operations. This allows us to perform any operation we desire in the normalizer of $\mathcal{G}$. Note that Sec. VI provides us with another way to get these operations. Having two methods available broadens the choices for picking the most efficient implementations.

In order to perform universal computation on the fivequbit code, we must know how to perform a Toffoli gate. Shor [7] gave a method for producing a Toffoli gate that relied on the ability to perform the gate

$$
|a\rangle|b\rangle|c\rangle \rightarrow(-1)^{a(b c)}|a\rangle|b\rangle|c\rangle,
$$

where $|a\rangle$ is either $|0 \cdots 0\rangle$ or $|1 \cdots 1\rangle$ and $|b\rangle$ and $|c\rangle$ are encoded 0 's or 1 's. For the codes Shor considered, this gate could be performed by applying it bitwise, because the conditional sign could be applied bitwise. All of the qubits in the first block are either 0 or 1 , so a controlled conditional sign from the first block will produce a conditional sign on the second two blocks whenever the first block is 1 .

For the five-qubit code, this gate is not quite as straightforward, but is still not difficult. To perform the two-qubit conditional sign gate on the five-qubit code, we need to perform a series of one- and three-qubit gates and measurements. However, if we perform each of these gates and measurements conditional on the value of $a$, we have performed the conditional sign gate on $|b\rangle|c\rangle$ if and only if the first block is 1 . To do this, we perform the part of each acting on the $i$ th qubit conditioned on the $i$ th qubit of $|a\rangle$. A single qubit error in $|a\rangle$ will therefore only result in a single qubit error in the state after the operation.

Performing measurements conditioned on $|a\rangle$ requires some care, but it can be done. The DiVincenzo and Shor method of performing a measurement is to perform a transversal CNOT to an ancilla in a superposition of all even parity states. The parity of the ancilla tells us the measurement result. If the ancilla instead begins in the superposition of all states, even and odd, performing the CNOTs and measuring the ancilla does nothing at all to the original state and gives no information. Therefore, to perform a measurement conditioned on the value of $a$, we should prepare the ancilla to hold the measurement result in the sum of even parity states when $a=1$ and the sum of all states when $a=0$. As with the usual measurement procedure, we must carefully verify this ancilla to avoid the possibility of correlated errors entering the data.

After this, the rest of Shor's construction of the Toffoli gate carries over straightforwardly. It involves a number of measurements and operations from the normalizer of $\mathcal{G}$. We have already discussed how to do all of those. The one remaining operation that is necessary is

$$
|a\rangle|d\rangle \rightarrow(-1)^{a d}|a\rangle|d\rangle
$$

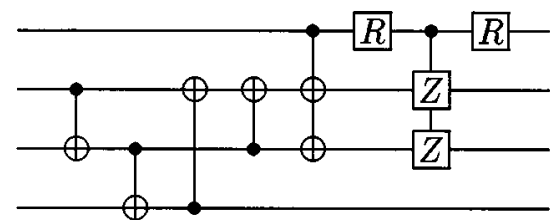

FIG. 2. Network to perform the four-qubit gate.

where $|d\rangle$ is an encoded state and $|a\rangle$ is again $|0 \cdots 0\rangle$ or $|1 \cdots 1\rangle$. However, this is just $\bar{Z}$ applied to $|d\rangle$ conditioned on the value of $a$, which we can do as before, using at most one two-qubit gate on each qubit in the block. Therefore, we can perform universal fault-tolerant computation on the fivequbit code.

Note that there was nothing particularly unique about the five-qubit code that made the construction of the Toffoli gate possible. The only property we needed was the ability to perform an encoded conditional sign gate.

\section{GATES FOR ANY STABILIZER CODE}

Consider the following transformation:

$$
\begin{aligned}
& X \otimes I \otimes I \otimes I \rightarrow X \otimes X \otimes X \otimes I, \\
& I \otimes X \otimes I \otimes I \rightarrow I \otimes X \otimes X \otimes X, \\
& I \otimes I \otimes X \otimes I \rightarrow X \otimes I \otimes X \otimes X, \\
& I \otimes I \otimes I \otimes X \rightarrow X \otimes X \otimes I \otimes X, \\
& Z \otimes I \otimes I \otimes I \rightarrow Z \otimes Z \otimes Z \otimes I, \\
& I \otimes Z \otimes I \otimes I \rightarrow I \otimes Z \otimes Z \otimes Z, \\
& I \otimes I \otimes Z \otimes I \rightarrow Z \otimes I \otimes Z \otimes Z, \\
& I \otimes I \otimes I \otimes Z \rightarrow Z \otimes Z \otimes I \otimes Z .
\end{aligned}
$$

A possible gate array to perform this operation is given in Fig. 2. This operation takes $M \otimes I \otimes I \otimes I$ to $M \otimes M \otimes M \otimes I$, and cyclic permutations of this, so if $M \in S$, the image of these operations is certainly in $S \times S \times S \times S$. This therefore is a valid transversal operation on any stabilizer code. The encoded operation it performs is just itself. There is a family of related operations for any even number of qubits (the twoqubit case is trivial), but we only need to concern ourselves with the four-qubit operation.

Suppose we have two data qubits. Prepare the third and fourth qubits in the state $|00\rangle$, apply the above transformation, and then measure $X$ for the third and fourth qubits. The resulting transformation on the first two qubits is then

$$
\begin{aligned}
& X \otimes I \rightarrow X \otimes X, \\
& I \otimes X \rightarrow I \otimes X, \\
& Z \otimes I \rightarrow Z \otimes I, \\
& I \otimes Z \rightarrow Z \otimes Z .
\end{aligned}
$$


This is precisely the controlled NOT. Since I showed in Sec. III that the CNOT was sufficient to get any operation in $N(\mathcal{G})$, we can get any such operation for any stabilizer code. In fact, using the Toffoli gate construction from Sec. V, we can perform universal computation.

Actually, this only gives universal computation for codes encoding a single qubit in a block, since if a block encodes multiple qubits, this operation performs the CNOT between corresponding encoded qubits in different blocks. To actually get universal computation, we will want to perform operations between qubits encoded in the same block. To do this, we need a few more tools, which will be presented in the next section. I will also consider a few more examples where we have tools beyond the ones available for any code.

\section{DISTANCE TWO CODES}

There is a large class of distance two codes with a very simple form. The stabilizer for these codes has just two generators, one a product of all $X$ 's and one a product of all $Z$ 's. The total number of qubits $n$ must be even. These codes encode $n-2$ qubits, and therefore serve as a good model for block codes encoding multiple qubits. While these distance two codes cannot actually correct a general error, they may be useful in their own right nonetheless. A distance two code can be used for error detection [17]. If we encode our computer using distance two codes, we will not be able to fix any errors that occur, but we will know if an error has invalidated our calculation. A better potential use of distance two codes is to fix located errors [18]. Suppose the dominant error source in our hardware comes from qubits leaving the normal computational space. In principle, without any coding, we can detect not only that this has happened, but in which qubit it has occurred. We can then use this information in conjunction with a distance two code to correct the state, as with a usual quantum error-correcting code. A final possible use of distance two codes is to concatenate them to produce codes that can correct multiple errors. Since the limiting factor in the computational threshold for concatenated codes is the time to do error correction, this offers potentially a great advantage. However, there is a significant complication in this program, since the codes given here encode more than one qubit, which complicates the concatenation procedure.

Because of the simple structure of these distance two codes, we can immediately see a number of possible faulttolerant operations. The bitwise Hadamard rotation and the bitwise CNOT are both permissible. If the total number of qubits is a multiple of 4 , the $P$ gate and the other single qubit operations are allowed, as well. What is less clear is how these various operations affect the encoded data.

The $\overline{X_{i}}$ operators for these codes are $X_{1} X_{i+1}$, where $i$ runs from 1 to $n-2$. The $\bar{Z}_{i}$ operators are $Z_{i+1} Z_{n}$. Therefore, swapping the $(i+1)$ th qubit with the $(j+1)$ th qubit will swap the $i$ th encoded qubit with the $j$ th encoded qubit. Swapping two qubits in a block is not a transversal operation, but if performed carefully, it can still be done faulttolerantly. One advantage of the swap operation is that any errors in one qubit will not propagate to the other, since they are swapped as well. However, applying the swap directly to the two qubits allows the possibility of an error in the swap gate itself producing errors in both qubits. We can circum- vent this by introducing a third ancilla qubit. Suppose we wish to swap A and B, which are in spots 1 and 2, using ancilla $\mathrm{C}$, in spot 3. First swap the qubits in spots 1 and 3, then 1 and 2, and finally 2 and 3 . Then A ends up in spot 2, B ends up in spot 1, and C ends up in spot 3, but A and B have never interacted directly. We would need two swap gates to go wrong in order to introduce errors to both $\mathrm{A}$ and B. Note that while the state $C$ does not matter, it should not be something important, since it is exposed to error from all three swap gates. Also note that we should perform error correction before interacting this block with another block, since errors could then spread between corresponding qubits, which have changed.

The action of the CNOT is simple. As for other CSS codes, it just produces a CNOT from each encoded qubits in the first block to the corresponding encoded qubit in the second block. The Hadamard rotation converts $\overline{X_{i}}$ to $Z_{1} Z_{i+1}$, which is equivalent (via multiplication by $M_{2}$ ) to $Z_{2} \cdots Z_{i} Z_{i+2} \cdots Z_{n}$. This is equal to $\overline{Z_{1}} \cdots \overline{Z_{i-1} Z_{i+1}} \cdots$ $\overline{Z_{n-2}}$. Similarly, $\overline{Z_{i}}$ becomes $\overline{X_{1}} \cdots \overline{X_{i-1} X_{i+1}} \cdots \overline{X_{n-2}}$. For instance, for the smallest case, $n=4$,

$$
\begin{aligned}
& \overline{X_{1}} \rightarrow \overline{Z_{2}}, \\
& \overline{Z_{1}} \rightarrow \overline{X_{2}}, \\
& \overline{X_{2}} \rightarrow \overline{Z_{1}}, \\
& \overline{Z_{2}} \rightarrow \overline{X_{1}} .
\end{aligned}
$$

The Hadamard rotation for $n=4$ performs a Hadamard rotation on each encoded qubit and simultaneously switches them. For larger $n$, it performs the Hadamard rotation on each qubit, and performs a variation of the class of codes discussed in Sec. VI.

For $n=4$, the $P$ gate acts as follows:

$$
\begin{gathered}
\overline{X_{1}} \rightarrow-Y_{1} Y_{2}=-\overline{X_{1} Z_{2}}, \\
\overline{X_{2}} \rightarrow-Y_{1} Y_{3}=-\overline{X_{2} Z_{1}}, \\
\overline{Z_{1}} \rightarrow \overline{Z_{1}}, \\
\overline{Z_{2}} \rightarrow \overline{Z_{2}} .
\end{gathered}
$$

A consideration of two-qubit gates allows us to identify this as a variant of the conditional sign gate. Specifically, this gate gives a sign of -1 unless both qubits are $|0\rangle$.

When we allow measurement, a trick becomes available that is useful for any multiple-qubit block code. Given one data qubit, prepare a second ancilla qubit in the state $|0\rangle$ $+|1\rangle$, then apply a CNOT from the second qubit to the first qubit and measure $Z$ for the first qubit. The initial stabilizer is $I \otimes X$; after the CNOT it is $X \otimes X$. Therefore the full operation takes $X \otimes I$ to $I \otimes X$ and $Z \otimes I$ to $Z \otimes Z$. We can discard the first qubit and the second qubit is in the initial data state. However, if we prepare the ancilla in the state $|0\rangle$, then apply a CNOT, the original state is unaffected. Therefore, by preparing a block with all but the $j$ th encoded qubit in the state $|0\rangle$, and with the $j$ th encoded qubit in the state $|0\rangle$ $+|1\rangle$, then applying a CNOT from the new block to a data 
TABLE III. The stabilizer and encoded $X$ 's and $Z$ 's for the eight-qubit code.

\begin{tabular}{lcccccccc}
\hline \hline$M_{1}$ & $X$ & $X$ & $X$ & $X$ & $X$ & $X$ & $X$ & $X$ \\
$M_{2}$ & $Z$ & $Z$ & $Z$ & $Z$ & $Z$ & $Z$ & $Z$ & $Z$ \\
$M_{3}$ & $X$ & $I$ & $X$ & $I$ & $Z$ & $Y$ & $Z$ & $Y$ \\
$M_{4}$ & $X$ & $I$ & $Y$ & $Z$ & $X$ & $I$ & $Y$ & $Z$ \\
$M_{5}$ & $X$ & $Z$ & $I$ & $Y$ & $I$ & $Y$ & $X$ & $Z$ \\
$\overline{X_{1}}$ & $X$ & $X$ & $I$ & $I$ & $I$ & $Z$ & $I$ & $Z$ \\
$\overline{X_{2}}$ & $X$ & $I$ & $X$ & $Z$ & $I$ & $I$ & $Z$ & $I$ \\
$\overline{X_{3}}$ & $X$ & $I$ & $I$ & $Z$ & $X$ & $Z$ & $I$ & $I$ \\
$\overline{Z_{1}}$ & $I$ & $Z$ & $I$ & $Z$ & $I$ & $Z$ & $I$ & $Z$ \\
$\overline{Z_{2}}$ & $I$ & $I$ & $Z$ & $Z$ & $I$ & $I$ & $Z$ & $Z$ \\
$\bar{Z}$ & $I$ & $I$ & $I$ & $I$ & $Z$ & $Z$ & $Z$ & $Z$ \\
\hline \hline
\end{tabular}

block and measuring the $j$ th encoded qubit in the data block, we can switch the $j$ th encoded qubit out of the data block and into the new, otherwise empty block.

This trick enables us to perform arbitrary operations on qubits from the same block for the distance two codes. We switch the qubits of interest into blocks of their own, use swap operations to move them into corresponding spots, then perform whole block operations to interact them. Then we can swap them back and switch them back into place in their original blocks.

The step that is missing for arbitrary stabilizer codes is the ability to move individual encoded qubits to different places within a block. Since the gate in Sec. VI gives us a block CNOT, we can perform the switching operation into an empty block. By using switching and whole block operations, we can perform an arbitrary one-qubit operation on any single encoded qubit within a block. The only remaining operation necessary is the ability to swap an encoded qubit from the $i$ th place to the $j$ th place. We can do this using quantum teleportation. All that is required is an otherwise empty block with the $i$ th and $j$ th encoded qubits in the entangled state $|00\rangle+|11\rangle$. Then we need only perform singlequbit operations and a CNOT between the qubits in the $i$ th places, both of which we can do. To prepare the entangled state, we simply start with the +1 -eigenstate of $\bar{Z}_{i}$ and $\overline{Z_{j}}$, then measure the eigenvalue of $\overline{X_{i} X_{j}}$ (and correct if the result is -1$)$. This is just an operator in $\mathcal{G}$, so we know how to do this. The state stays in an eigenvector of $\overline{Z_{i} Z_{j}}$, which commutes with $\overline{X_{i} X_{j}}$, so the result will be the desired encoded Bell state. We can then teleport the $i$ th qubit in one otherwise empty block to the $j$ th qubit in the block originally containing the Bell state. This was all we needed to allow universal computation on any stabilizer code.

\section{THE EIGHT QUBIT CODE}

There is a code correcting one error encoding three qubits in eight qubits $[4,5,19]$. The stabilizer is given in Table III. There are no transversal operations that leave this stabilizer fixed except the automatic ones in $N(S)$. However, when we allow swaps between the constituent qubits, a number of possibilities become available.

One possible operation is to swap the first four qubits with the second four qubits. This leaves $M_{1}, M_{2}$, and $M_{4}$ un- changed. $M_{3}$ becomes instead $M_{1} M_{2} M_{3}$, and $M_{5}$ becomes $M_{1} M_{5}$. On the encoded qubits, this induces the transformation

$$
\begin{aligned}
& X \otimes I \otimes I \rightarrow X \otimes I \otimes Z, \\
& I \otimes X \otimes I \rightarrow I \otimes X \otimes I, \\
& I \otimes I \otimes X \rightarrow Z \otimes I \otimes X, \\
& Z \otimes I \otimes I \rightarrow Z \otimes I \otimes I, \\
& I \otimes Z \otimes I \rightarrow I \otimes Z \otimes I, \\
& I \otimes I \otimes Z \rightarrow I \otimes I \otimes Z .
\end{aligned}
$$

This is just a conditional sign on the first and third qubits, with the second encoded qubit unaffected. Through singlequbit transformations, we can convert this to a controlled NOT, and using this perform a swap between the first and third encoded positions.

Another operation is to swap qubits one and two with three and four and qubits five and six with seven and eight. This leaves $M_{1}, M_{2}$, and $M_{3}$ unchanged, and converts $M_{4}$ to $M_{2} M_{4}$ and $M_{5}$ to $M_{1} M_{5}$. On the encoded qubits, it induces the transformation

$$
\begin{gathered}
X \otimes I \otimes I \rightarrow X \otimes Z \otimes Z, \\
I \otimes X \otimes I \rightarrow Z \otimes X \otimes Z, \\
I \otimes I \otimes X \rightarrow Z \otimes Z \otimes X, \\
Z \otimes I \otimes I \rightarrow Z \otimes I \otimes I, \\
I \otimes Z \otimes I \rightarrow I \otimes Z \otimes I, \\
I \otimes I \otimes Z \rightarrow I \otimes I \otimes Z .
\end{gathered}
$$

We could also switch the odd numbered qubits with the even numbered qubits. That leaves $M_{1}$ and $M_{2}$ unchanged, while turning $M_{3}$ into $M_{1} M_{3}, M_{4}$ into $M_{1} M_{4}$, and $M_{5}$ into $M_{1} M_{2} M_{5}$. On the encoded qubits it induces

$$
\begin{gathered}
X \otimes I \otimes I \rightarrow X \otimes I \otimes Z, \\
I \otimes X \otimes I \rightarrow I \otimes X \otimes Z, \\
I \otimes I \otimes X \rightarrow Z \otimes Z \otimes X, \\
Z \otimes I \otimes I \rightarrow Z \otimes I \otimes I, \\
I \otimes Z \otimes I \rightarrow I \otimes Z \otimes I, \\
I \otimes I \otimes Z \rightarrow I \otimes I \otimes Z .
\end{gathered}
$$

This is just a conditional sign between the first and third places followed by a conditional sign between the second and third places. Combined with the first operation, it gives us a conditional sign between the second and third places, which we can again convert to a swap between the second 
and third encoded positions. This allows us to swap any two encoded qubits in the block, which is sufficient to give us universal computation.

In this case, the symmetries of the code naturally became allowed transformations of the stabilizer. This is likely to hold true in many other cases as well. As with the five-qubit code, we now have two protocols for universal computation, but multiple methods again allow us more freedom in choosing efficient methods.

\section{SUMMARY AND DISCUSSION}

I have presented a general theory for understanding when it is possible to apply a given operation transversally to a given quantum error-correcting code, and for understanding the results of making a measurement on a stabilizer code. These results clarify the advantages of the doubly even selfdual CSS codes used by Shor [7]. They also provide protocols for performing universal computation on any stabilizer code. In many cases, the protocols described here call for a number of steps to perform most simple operations, so more efficient protocols for specific codes are desirable, and I expect the methods described in this paper will be quite helpful when searching for these protocols.

Efficient use of space is also important. Existing methods of fault-tolerant computation use space very inefficiently, and being able to use more efficient codes (such as those encoding multiple qubits in a block) could be very helpful in reducing the space requirements.

\section{ACKNOWLEDGMENTS}

This work was supported in part by the U.S. Department of Energy under Grant No. DE-FG03-92-ER40701 and by DARPA under Grant No. DAAH04-96-1-0386 administered by the Army Research Office. I would like to thank John Preskill, Manny Knill, Richard Cleve, and David DiVincenzo for helpful discussions.

\section{APPENDIX: PROOF THAT $R, P$, AND CNOT GENERATE $N(\mathcal{G})$}

I claimed before that products of $R, P$, and CNOT actually gave us all of the elements of $N(\mathcal{G})$. The proof in this appendix provides a neat application of the formalism presented in Secs. II and III. The proof is constructive, and is quite useful for finding networks to perform multiple-qubit operations in $N(\mathcal{G})$. The one-qubit operations in $N(\mathcal{G})$ correspond to the six automorphisms of $D_{4}$ given by $R, P, Q, T$, $T^{2}$, and of course the identity. We have already seen that $Q=P^{\dagger} R P^{\dagger}$. Also, $T=P Q^{\dagger}$, so all one-qubit operations are covered.

We can also perform all two-qubit operations. Every automorphism of $D_{4} \times D_{4}$ can be produced by a composition of controlled NOT and single-qubit operations. For instance, take

$$
Z \otimes I \rightarrow X \otimes X
$$$$
I \otimes Z \rightarrow Z \otimes Z
$$

$$
\begin{aligned}
& X \otimes I \rightarrow i Y \otimes X, \\
& I \otimes X \rightarrow i Z \otimes Y .
\end{aligned}
$$

This permutation can be produced by performing the cyclic permutation $X \rightarrow i Y \rightarrow Z \rightarrow X$ on the first qubit and a phase rotation $X \rightarrow i Y$ on the second qubit, and then performing a standard controlled NOT from the first qubit to the second qubit. It is straightforward to consider the other possibilities and show that they too can be written using a CNOT and one-qubit gates.

I will show that the larger gates can be made this way by induction on the number of qubits. Suppose we know this to be true for all $n$-qubit gates, and we have an $(n+1)$-qubit gate $U$. On an arbitrary input state $|0\rangle|\psi\rangle+|1\rangle|\phi\rangle$ (where $|\psi\rangle$ and $|\phi\rangle$ are $n$-qubit states), the output state will be

$$
\left(|0\rangle\left|\psi_{1}\right\rangle+|1\rangle\left|\psi_{2}\right\rangle\right)+\left(|0\rangle\left|\phi_{1}\right\rangle+|1\rangle\left|\phi_{2}\right\rangle\right)
$$

Suppose that under the applied transformation, $M=U(Z \otimes I$ $\otimes \cdots \otimes I) U^{\dagger}$ anticommutes with $Z \otimes I \otimes \cdots \otimes I$. If it does not, we can apply a one-qubit transformation and/or rearrange qubits so that $M=X \otimes M^{\prime}$, where $M^{\prime}$ is an $n$-qubit operation. Suppose we apply $U$ to $|0\rangle|\psi\rangle$. If we were then to measure $Z$ for the first qubit, we would get either 0 , in which case the other qubits are in state $\left|\psi_{1}\right\rangle$, or 1 , in which case the remaining qubits are in state $\left|\psi_{2}\right\rangle$. The above analysis of measurements shows that $\left|\psi_{1}\right\rangle$ and $\left|\psi_{2}\right\rangle$ are therefore related by the application of $M^{\prime}$. Define $U^{\prime}$ by $U^{\prime}|\psi\rangle=\left|\psi_{1}\right\rangle$. Then

$$
U(|0\rangle|\psi\rangle)=(I+M)\left(|0\rangle \otimes U^{\prime}|\psi\rangle\right)
$$

Let $N=U(X \otimes I \otimes \cdots \otimes I) U^{\dagger}$. Again, we can apply a onequbit operation so that either $N=Z \otimes N^{\prime}$ or $N=I \otimes N^{\prime}$. We can always put $M$ and $N$ in their required forms simultaneously. Then

$$
\begin{aligned}
U(|1\rangle|\phi\rangle) & =N U(|0\rangle|\phi\rangle) \\
& =N(I+M)\left(|0\rangle \otimes U^{\prime}|\phi\rangle\right) \\
& =(I-M) N\left(|0\rangle \otimes U^{\prime}|\phi\rangle\right) \\
& =(I-M)\left(|0\rangle \otimes N^{\prime} U^{\prime}|\phi\rangle\right),
\end{aligned}
$$

using the above form of $N$ and the fact that $\{M, N\}=0$.

Now, $U^{\prime}$ is an $n$-qubit operation, so we can build it out of $R, P$, and CNOT. To apply $U$, first apply $U^{\prime}$ to the last $n$ qubits. Now apply $N^{\prime}$ to the last $n$ qubits conditioned on the first qubit being 1 . We can do this with just a series of CNOTs and one-qubit operations. Now apply a Hadamard transform to the first qubit. This puts the system in the state

$$
(|0\rangle+|1\rangle) \otimes U^{\prime}|\psi\rangle+(|0\rangle-|1\rangle) \otimes N^{\prime} U^{\prime}|\phi\rangle .
$$


Now, apply $M^{\prime}$ to the last $n$ qubits conditioned on the first qubit. Again, we can do this with just CNOTs and one-qubit operations. This leaves the system in the state

$$
\begin{aligned}
& |0\rangle \otimes U^{\prime}|\psi\rangle+|1\rangle \otimes M^{\prime} U^{\prime}|\psi\rangle+|0\rangle \otimes N^{\prime} U^{\prime}|\phi\rangle \\
& -|1\rangle \otimes M^{\prime} N^{\prime} U^{\prime}|\phi\rangle,
\end{aligned}
$$

$$
\begin{aligned}
= & |0\rangle \otimes U^{\prime}|\psi\rangle+M\left(|0\rangle \otimes U^{\prime}|\psi\rangle\right)+|0\rangle \otimes N^{\prime} U^{\prime}|\phi\rangle \\
& \quad-M\left(|0\rangle \otimes N^{\prime} U^{\prime}|\phi\rangle\right) \\
= & (I+M)\left(|0\rangle \otimes U^{\prime}|\psi\rangle\right)+(I-M)\left(|0\rangle \otimes N^{\prime} U^{\prime}|\phi\rangle\right),
\end{aligned}
$$

which we can recognize as the desired end state after applying $U$.
[1] P. W. Shor, Phys. Rev. A 52, 2493 (1995).

[2] A. R. Calderbank and P. W. Shor, Phys. Rev. A 54, 1098 (1996).

[3] A. Steane, Proc. R. Soc. London, Ser. A 452, 2551 (1996).

[4] D. Gottesman, Phys. Rev. A 54, 1862 (1996).

[5] A. R. Calderbank, E. M. Rains, P. W. Shor, and N. J. A. Sloane, Phys. Rev. Lett. 78, 405 (1997).

[6] A. R. Calderbank, E. M. Rains, P. W. Shor, and N. J. A. Sloane, IEEE Trans. Inf. Theory (to be published).

[7] P. W. Shor, in Proceedings of the 37th Symposium on Foundations of Computer Science (IEEE Computer Society Press, Los Alamitos, CA, 1996), p. 56.

[8] E. Knill, R. Laflamme, and W. Zurek, e-print quant-ph/9610011.

[9] D. Aharonov and M. Ben-Or, e-print quant-ph/9611025.
[10] D. DiVincenzo and P. W. Shor, Phys. Rev. Lett. 77, 3260 (1996).

[11] C. H. Bennett, D. P. DiVincenzo, J. A. Smolin, and W. K. Wootters, Phys. Rev. A 54, 3824 (1996).

[12] E. Knill, e-print quant-ph/9608049.

[13] E. Knill (private communication).

[14] R. Cleve and D. Gottesman, Phys. Rev. A 56, 76 (1997).

[15] R. Laflamme, C. Miquel, J. P. Paz, and W. H. Zurek, Phys. Rev. Lett. 77, 198 (1996).

[16] E. Knill and R. Laflamme, e-print quant-ph/9608012.

[17] L. Vaidman, L. Goldenberg, and S. Wiesner, Phys. Rev. A 54, R1745 (1996).

[18] M. Grassl, T. Beth, and T. Pellizzari, Phys. Rev. A 56, 33 (1997).

[19] A. Steane, Phys. Rev. A 54, 4741 (1996). 